\title{
Age itself or age-associated comorbidities? A nationwide analysis of outcomes of geriatric trauma
}

\author{
Chih-Yuan Fu ${ }^{1,2}$. Francesco Bajani ${ }^{1} \cdot$ Marissa Bokhari ${ }^{1} \cdot$ Frederick Starr $^{1} \cdot$ Thomas Messer $^{1} \cdot$ Matthew Kaminsky $^{1}$. \\ Andrew Dennis ${ }^{1} \cdot$ Victoria Schlanser $^{1} \cdot$ Justin Mis $^{1} \cdot$ Stathis Poulakidas ${ }^{1} \cdot$ Faran Bokhari ${ }^{1}$
}

Received: 31 August 2020 / Accepted: 27 December 2020 / Published online: 27 January 2021

(c) Springer-Verlag GmbH, DE part of Springer Nature 2021

\begin{abstract}
Purpose Geriatric trauma patients present physiological challenges to care providers. A nationwide analysis was performed to evaluate the roles of age alone versus age-associated comorbidities in the morbidity and mortality of elderly patients with blunt abdominal trauma (BAT).

Methods Patients with BAT registered in the National Trauma Data Bank from 2013 to 2015 were analyzed using propensity score matching (PSM) to evaluate the mortality rate, complication rate, hospital length of stay (LOS), intensive care unit (ICU) LOS and ventilator days between young (age $<65$ ) and elderly (age $\geq 65$ ) patients. An adjusted multivariate logistic regression (MLR) model was also used to evaluate the effect of age itself and age-associated comorbidities on mortality. Results There were 41,880 patients with BAT during the study period. In elderly patients, the injury severity score (ISS) decreased with age, but the mortality rate increased inversely (from 5.0 to $13.5 \%$ ). Under a similar condition and proportion of age-associated comorbidities after a well-batched PSM analysis, elderly patients had significantly higher mortality rates ( $8.0 \%$ vs. $1.9 \%, p<0.001)$, higher complication rates ( $35.1 \%$ vs. $30.6 \%, p<0.001)$, longer hospital LOS ( 8.9 vs. 8.1 days, $p<0.001)$, longer ICU LOS (3.7 vs. 2.7 days, $p<0.001)$ and more ventilator days ( 1.1 vs. 0.5 days, $p<0.001)$ than young patients. Furthermore, the MLR analysis showed that age itself served as an independent factor for mortality (odds ratio: $1.049,95 \%$ CI 1.043-1.055, $p<0.001)$, but age-associated comorbidity was not.

Conclusion In patients with BAT, age itself appeared to have an independent and deleterious effect on mortality, but ageassociated comorbidity did not.
\end{abstract}

Keywords Geriatric trauma $\cdot$ Blunt abdominal trauma $\cdot$ Age-associated comorbidity

The paper was presented in the XXXI Pan-American Congress of Trauma, Critical Care \& Emergency Surgery \& XLIV National Congress Advances in Surgery, August 14-17, 2018 in Cartagena, Colombia (ID \#ILAB-8).

Supplementary Information The online version contains supplementary material available at https://doi.org/10.1007/s0006 8-020-01595-8.

Faran Bokhari

fbokhari@cookcountyhhs.org

1 Department of Trauma and Burn Surgery, Stroger Hospital of Cook County, Rush University, 8th floor, 1950 West Polk Street, Chicago, IL 60612, USA

2 Department of Trauma and Emergency Surgery, Chang Gung Memorial Hospital, No.5 Fu Shihg St., Taoyuan 333, Taiwan

\section{Introduction}

Developed nations have an aging population. In the United States (US), persons 65 years and over account for $14 \%$ of all trauma patients [1]. Compared with younger patients, geriatric patients sustain less severe traumatic injuries. However, elderly trauma patients face an increased risk for adverse outcomes after injury due to a progressive decline in physiological function [2-4]. Therefore, geriatric patients represent an increasing number and proportion of trauma admissions and deaths. The management of geriatric trauma is a major medical and socioeconomic issue.

Recent reports have indicated that aging is not only a natural process but also a disease [5, 6]. However, elderly patients also have more age-associated comorbidities than young patients. Therefore, it is controversial whether aging is a natural and physiological process or whether it 
represents a pathology. The role of age alone versus ageassociated comorbidities in the morbidity and mortality of elderly patients with trauma is not clear.

Compared with penetrating trauma, blunt abdominal trauma (BAT) is more common in elderly patients. The management of BAT is complicated. In addition to resuscitation in the emergency department (ED), surgical operations and intensive critical care might be needed [7, 8]. Physicians face challenges in the management of abdominal traumarelated complications and secondary systemic complications due to a poor physiologic capacity to respond to injury in elderly patients. Special care is needed for their degenerated anatomy and physiology and decreased tolerance to traumarelated stresses.

In the current study, a nationwide analysis was performed to delineate the characteristics and epidemiology of elderly patients with BAT. We hypothesized that age itself and ageassociated comorbidities independently affect the outcomes of elderly patients with BAT.

\section{Methods}

\section{Study design and setting}

The National Trauma Data Bank (NTDB) was queried from July 1, 2012, to June 30, 2015 [9]. The inclusion criteria were patients with BAT (trauma mechanism: blunt, origin file: RDS_ECODE and RDS_ECODEDES; ICD-9-CM: 863. xx-869.xx, origin file: RDS_DCODE and RDS_DCODEDES) (Electronic Supplementary Material Table 1). Patients with burns, penetrating trauma, unknown trauma mechanism, concomitant head injury, and an incomplete record of age were excluded (Fig. 1).

Data for age, sex (origin file: RDS_DEMO), systolic blood pressure (SBP) in the ED, pulse in the ED, respiratory rate (RR) in the ED, Glasgow coma scale (GCS) in the ED (origin file: RDS_ED), transportation time (EMSMINS in origin file: RDS_ED), ED time (EDMINS in origin file: RDS_ED), use of transfusion (PCODEs: 99.0-99.09, origin file: RDS_PCODE and RDS_PCODEDES), abdominal surgery (origin file: RDS_PCODE and RDS_PCODEDES, PCODE in Electronic Supplementary Material Table 2) and injury severity score (ISS) were collected and evaluated. Twenty-five comorbidities were collected and recorded by the NTDB (origin file: RDS_COMORBID). Congestive heart failure $(\mathrm{CHF})(\mathrm{COMORKEY}=7)$, chronic obstructive pulmonary disease $(\mathrm{COPD})(\mathrm{COMORKEY}=23)$, cerebrovascular accident $(\mathrm{CVA})(\mathrm{COMORKEY}=10)$, dementia $($ COMORKEY $=26)$, diabetes mellitus $($ COMORKEY $=11)$, history of angina within 30 days (COMORKEY $=16$ ), history of myocardial infarction (COMORKEY $=17$ ), hypertension requiring medication $($ COMORKEY $=19)$ and

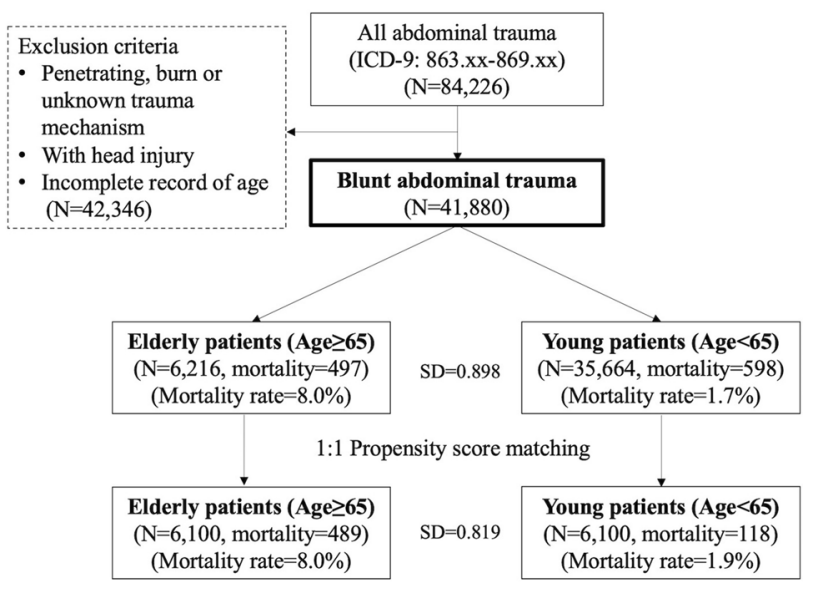

ICD-9=International classification of diseases, $\mathrm{SBP}=$ systolic blood pressure, $\mathrm{RR}=$-respiratory rate, $\mathrm{GCS}=$ Glasgow coma scale, $\mathrm{ISS}=$ =injury severity score, $\mathrm{SD}=$ standardized difference

Fig. 1 Study population and protocol of the current study

steroid use (COMORKEY $=24)$ were selected for analysis, because these comorbidities are common in elderly patients and may affect trauma outcomes [10-12].

Patients who were coded as deceased/expired in the HOSDISP column (hospital disposition) were defined as such (origin file: RDS_DISCHARGE). Per the NTDB, complications were defined as pneumonia, an unplanned return to the ICU, stroke, urinary tract injury, acute kidney injury, decubitus ulcer, pulmonary embolism, acute lung injury, severe sepsis, deep vein thrombosis, cardiac arrest with resuscitative efforts by the healthcare provider, drug or alcohol withdrawal syndrome, unplanned return to the operation room, organ/space surgical site infection, deep surgical site infection, myocardial infarction, catheter-related blood stream infection, osteomyelitis, extremity compartment syndrome, superficial surgical site infection and graft/prosthesis/flap failure (origin file: RDS_COMPLIC). Mortality, complications, hospital length of stay (LOS), intensive care unit (ICU) LOS and ventilator days (origin file: RDS_DISCHARGE) were designated as outcomes.

\section{Statistical analysis}

In the current study, three analytic approaches were utilized to evaluate the effect of age itself and age-associated comorbidities on outcomes for patients with BAT. First, we used a one-to-one propensity score matching (PSM) methodology to minimize the selection bias between young (age $<65$ ) and elderly patients (age $\geq 65$ ). Then, the greedy neighbor approach was used to construct pairs of young and elderly patients [13]. Covariables that may affect trauma outcomes clinically were put into PSM. These covariables included sex (male sex), SBP, pulse, RR, GCS, transportation time, 
ED time, age-associated comorbidities, abdominal surgery, use of transfusion, and ISS. A caliper was set as $0.1[14,15]$. Balanced matching was confirmed with standardized differences (SDs). When the SD was less than 0.1, the matching result could be considered balanced (Fig. 1 and upper area of Table 1) [16]. After a well-balanced matching, mortality, complications, hospital LOS, ICU LOS and ventilator days between young and elderly patients were compared (lower area of Table 1). A percentage with a 95\% confidence interval (CI) was presented for nominal data, and they were compared using the chi-square test. The mean with a $95 \%$ CI (SBP, pulse, RR, GCS and ISS are presented as the median and interquartile range) were presented for numerical data, and Student's $t$ test was used for comparisons. Statistical significance was considered when a $p$-value was less than 0.05 .

Second, an adjusted multivariate logistic regression (MLR) model was performed to evaluate the effect of age itself and age-associated comorbidities on mortality for patients with BAT (Table 2) [17]. The independent risk factors and the associated odds ratios for mortality of patients with BAT were analyzed accordingly.

Third, a subset analysis of elderly patients only was performed to evaluate the effect of age and age-associated comorbidities on mortality. The mortality rate was compared between elderly patients with and without age-associated comorbidities (Table 3). Furthermore, the role of age and age-associated comorbidities in mortality was evaluated using an MLR analysis in these elderly patients (Table 4).

We used R software, version 3.5.0 of R Core Team (R Foundation for Statistical Computing, Vienna, Austria, 2018) and R Studio software, version 1.1.453 of R Studio: Integrated Development for R (R Studio, Inc., Boston, Massachusetts, 2016) to merge and analyze original files of NTDB [18]. The current study was approved by

Table 1 Characteristics and outcomes of elderly and young patients with BAT

\begin{tabular}{|c|c|c|c|c|c|c|}
\hline \multirow[t]{2}{*}{ Characteristics } & \multicolumn{3}{|c|}{ Before propensity matching } & \multicolumn{3}{|c|}{ After propensity matching } \\
\hline & $\begin{array}{l}\text { Elderly patients } \\
(N=6216)\end{array}$ & $\begin{array}{l}\text { Young patients } \\
(N=35,664)\end{array}$ & $\mathrm{SD}^{\mathrm{a}}$ & $\begin{array}{l}\text { Elderly patients } \\
(N=6100)\end{array}$ & $\begin{array}{l}\text { Young patients } \\
(N=6100)\end{array}$ & SD \\
\hline Age (years) & $75.1(75.0-75.3)$ & $33.9(33.8-34.1)$ & 2.764 & $75.1(74.9-75.3)$ & $38.9(38.5-39.3)$ & 3.037 \\
\hline Male $(\%)$ & $57.3(56.1-58.5)$ & $68.0(67.5-68.5)$ & 0.255 & $58.0(56.8-59.2)$ & $59.3(58.1-60.5)$ & 0.028 \\
\hline $\mathrm{SBP}$ in $\mathrm{ED}(\mathrm{mmHg})$ & $132(41)$ & $125(29)$ & 0.237 & $132(41)$ & $130(31)$ & 0.018 \\
\hline Pulse in ED (/min) & $83(25)$ & $92(29)$ & 0.412 & $83(25)$ & $86(26)$ & 0.064 \\
\hline $\mathrm{RR}$ in $\mathrm{ED}(/ \mathrm{min})$ & $18(4)$ & $18(6)$ & 0.081 & $18(4)$ & $18(4)$ & 0.002 \\
\hline GCS in ED & $15(0)$ & $15(0)$ & 0.016 & $15(0)$ & $15(0)$ & 0.005 \\
\hline $\begin{array}{l}\text { Transportation time } \\
\text { (min) }\end{array}$ & $329.8(311.8-347.9)$ & $301.5(294.1-309.0)$ & 0.040 & $328.4(310.4-346.5)$ & $333.1(313.4-352.8)$ & 0.006 \\
\hline ED time (min) & $288.3(280.7-295.8)$ & $260.1(256.9-263.4)$ & 0.090 & $288.4(280.7-296.0)$ & $269.9(262.1-277.7)$ & 0.060 \\
\hline $\begin{array}{l}\text { Age-associated comor- } \\
\text { bidity }(\%)\end{array}$ & $79.6(78.6-80.6)$ & $47.3(46.8-47.8)$ & 0.808 & $79.2(78.2-80.2)$ & $79.0(78.0-80.0)$ & 0.006 \\
\hline Abdominal surgery (\%) & $15.5(14.6-16.4)$ & $19.4(19.0-19.8)$ & 0.146 & $15.6(14.7-16.5)$ & $17.8(16.8-18.8)$ & 0.085 \\
\hline Use of transfusion (\%) & $28.4(27.3-9.5)$ & $17.0(16.6-17.4)$ & 0.363 & $27.5(26.4-28.6)$ & $29.4(28.3-30.5)$ & 0.052 \\
\hline ISS & $13(10)$ & $14(12)$ & 0.123 & $13(10)$ & $13(12)$ & 0.042 \\
\hline Outcomes & & & $p$-value & & & $p$-value \\
\hline Hospital LOS (day) & $8.9(8.6-9.1)$ & $7.8(7.7-7.9)$ & $<0.001^{\dagger}$ & $8.9(8.6-9.1)$ & $8.1(7.9-8.3)$ & $<0.001^{\dagger}$ \\
\hline ICU LOS (day) & $3.7(3.5-3.8)$ & $2.5(2.4-2.5)$ & $<0.001^{\dagger}$ & $3.7(3.5-3.9)$ & $2.7(2.5-2.9)$ & $<0.001^{\dagger}$ \\
\hline Ventilator days & $1.1(0.9-1.2)$ & $0.3(0.3-0.4)$ & $<0.001^{\dagger}$ & $1.1(0.9-1.2)$ & $0.5(0.4-0.7)$ & $<0.001^{\dagger}$ \\
\hline Complication rate $(\%)$ & $35.2(34.0-36.4)$ & $26.2(25.7-26.7)$ & $<0.001^{\ddagger}$ & $35.1(33.9-36.3)$ & $30.6(29.4-31.8)$ & $<0.001^{\ddagger}$ \\
\hline Mortality rate (\%) & $8.0(7.3-8.7)$ & $1.7(1.6-1.8)$ & $<0.001^{\ddagger}$ & $8.0(7.3-8.7)$ & $1.9(1.6-2.0)$ & $<0.001^{t}$ \\
\hline
\end{tabular}

Both before propensity matching $(N=41,880)$ and after propensity matching $(N=12,200)$ are shown

Numerical data: mean (95\% CI lower-upper) (SBP in ED, pulse in ED, RR in ED, GCS in ED and ISS were presented as the median and interquartile range)

Nominal data: percentage (95\% CI lower-upper)

$B A T$ blunt abdominal trauma, $S B P$ systolic blood pressure, $R R$ respiratory rate, $G C S$ Glasgow coma scale, $E D$ emergency department, $I S S$ injury severity score, $L O S$ length of stay, ICU intensive care unit, $C I$ confidence interval

†Student's $t$ test

${ }^{\ddagger}$ Chi-square test

${ }^{a} \mathrm{SD}=$ standardized difference ( $\mathrm{SD} \geq 0.1$ represent significant differences in covariables between groups) 
Table 2 Multivariate logistic regression analysis for the evaluation of independent risk factors for mortality in patients with BAT

\begin{tabular}{lrlll}
\hline Variables & $p$-value* & Odds of mortality & \multicolumn{2}{l}{$95 \%$ CI } \\
\cline { 3 - 5 } & & & Lower & Upper \\
\hline Age & $<0.001$ & 1.049 & 1.043 & 1.055 \\
$\begin{array}{l}\text { Elderly patient } \\
\quad \text { age } \geq 65)\end{array}$ & $<0.001$ & 1.591 & 1.268 & 1.995 \\
Male & & & & \\
SBP in ED (mmHg) & $<0.001$ & 0.993 & 1.116 & 1.483 \\
Pulse in ED (/min) & $<0.001$ & 1.010 & 0.991 & 0.994 \\
RR in ED (/min) & 0.771 & - & 1.007 & 1.012 \\
GCS in ED & $<0.001$ & 0.902 & - & - \\
Transportation time & 0.058 & - & 0.891 & 0.913 \\
$\quad($ min) & & & - & - \\
ED time (min) & 0.043 & 1.000 & & \\
ISS & $<0.001$ & 1.051 & 0.999 & 1.000 \\
Age-associated comor- & 0.494 & - & 1.045 & 1.056 \\
$\quad$ bidity & & & - & - \\
Abdominal operation & $<0.001$ & 3.028 & 2.618 & 3.503 \\
Use of transfusion & $<0.001$ & 2.104 & 1.826 & 2.425 \\
Constant & $<0.001$ & - & - & - \\
\hline
\end{tabular}

$B A T$ blunt abdominal trauma, $S B P$ systolic blood pressure, $E D$ emergency department, $R R$ respiratory rate, GCS Glasgow coma scale, ISS injury severity score, $C I$ confidence interval

The odds of mortality and 95\% CI are not shown for insignificant variables $(p>0.05)$

*Multivariate logistic regression

the institutional research board of our institution (Ref No.
18-068X).

\section{Results}

\section{Study population}

From July 1, 2012 to June 30, 2015, there were 84,226 abdominal trauma patients (ICD-9: 863.xx-869.xx) in the NTDB. A total of 41,880 patients with BAT with a mean age of 40.1 years were studied. A total of $6216(14.8 \%)$ of the patients were elderly (age $\geq 65$ ) (Fig. 1). Figure 2 presents the patient distribution among ages (lower area), the relationship between the ISS and age (middle area) and the relationship between the mortality rate and age (upper area). The patient distributions showed that most patients with BAT were 16-30 years old (16-20: $N=4606,11.0 \%$; 21-25: $N=4854,11.6 \% ; 26-30: N=3741,8.9 \%$ ). In elderly patients, the number of BATs gradually decreased with age. There was an inverse relationship between age and ISS in young and elderly patients. Although the ISS decreased with age in elderly patients (median from 14 to 12), the mortality rate increased inversely (from 5.0 to $13.5 \%$ ).

\section{PSM and the MLR cohort analysis}

PSM yielded well-balanced cohorts of 12,200 patients from 41,880 patients with BAT (Table 1). After matching, elderly patients had significantly higher mortality rates $(8.0 \%$ vs. $1.9 \%, p<0.001)$, higher complication rates $(35.1 \%$ vs.
Table 3 Comparisons of elderly BAT patients with and without age-associated comorbidities $(N=6216)$

\begin{tabular}{lllr}
\hline Variables & $\begin{array}{l}\text { With age-associated comor- } \\
\text { bidities }(N=4945)\end{array}$ & $\begin{array}{l}\text { Without age-associated } \\
\text { comorbidities }(N=1271)\end{array}$ & $p$ value \\
\hline Age & $75.4(75.2-75.6)$ & $73.9(73.5-74.3)$ & $<0.001^{*}$ \\
Male (\%) & $57.6(56.2-59.0)$ & $55.9(53.2-58.6)$ & $0.260^{\dagger}$ \\
SBP in ED (mmHg) & $132(42)$ & $131(39)$ & $0.326^{*}$ \\
Pulse in ED (/min) & $83(25)$ & $83(25)$ & $0.826^{*}$ \\
RR in ED (/min) & $18(4)$ & $18(4)$ & $0.457^{*}$ \\
GCS in ED & $15(0)$ & $15(0)$ & $0.435^{*}$ \\
Transportation time (min) & $332.4(312.4-352.5)$ & $319.7(278.0-361.3)$ & $0.576^{*}$ \\
ED time (min) & $291.1(282.5-299.6)$ & $277.2(261.2-293.3)$ & $0.147^{*}$ \\
Abdominal surgery (\%) & $15.5(14.5-16.5)$ & $15.8(13.8-17.8)$ & $0.763^{\dagger}$ \\
Use of transfusion $(\%)$ & $30.1(28.8-31.4)$ & $22.0(19.7-22.3)$ & $<0.001^{\dagger}$ \\
ISS & $13(10)$ & $14(10)$ & $<0.001^{*}$ \\
Mortality $(\%)$ & $8.2(7.4-9.0)$ & $7.1(5.7-8.5)$ & $0.178^{\dagger}$ \\
\hline
\end{tabular}

Numerical data: mean (95\% CI lower-upper) (SBP in ED, pulse in ED, RR in ED, GCS in ED and ISS were presented as the median and interquartile range)

Nominal data: percentage (95\% CI lower-upper)

$B A T$ blunt abdominal trauma, $S B P$ systolic blood pressure, $R R$ respiratory rate, GCS Glasgow coma scale, $E D$ emergency department, ISS injury severity score, $C I$ confidence interval

* Student $T$ test

${ }^{\dagger}$ Chi-square test 
Table 4 Multivariate logistic regression analysis for the evaluation of independent risk factors for mortality in elderly BAT patients $(N=6216)$

\begin{tabular}{lrlll}
\hline Variables & $p$ value $^{\dagger}$ & Odds of mortality & \multicolumn{2}{l}{$95 \%$ CI } \\
\cline { 3 - 5 } & & & Lower & Upper \\
\hline Age & $<0.001$ & 1.076 & 1.060 & 1.092 \\
Male & $<0.001$ & 1.604 & 1.299 & 1.980 \\
SBP in ED (mmHg) & $<0.001$ & 0.994 & 0.991 & 0.997 \\
Pulse in ED (/min) & $<0.001$ & 1.012 & 1.008 & 1.016 \\
RR in ED (/min) & 0.431 & - & - & - \\
GCS in ED & $<0.001$ & 0.925 & 0.906 & 0.944 \\
Transportation time & 0.338 & - & - & - \\
$\quad$ (min) & & & & \\
ED time (min) & 0.212 & - & - & - \\
ISS & $<0.001$ & 1.061 & 1.051 & 1.071 \\
Age-associated comor- & 0.090 & - & - & - \\
$\quad$ bidity & & & & \\
Abdominal operation & $<0.001$ & 2.755 & 2.193 & 3.461 \\
Use of transfusion & $<0.001$ & 1.809 & 1.462 & 2.239 \\
Constant & $<0.001$ & - & - & - \\
\hline
\end{tabular}

$B A T$ blunt abdominal trauma, $S B P$ systolic blood pressure, $E D$ emergency department, $R R$ respiratory rate, GCS Glasgow coma scale, ISS injury severity score, $C I$ confidence interval

The odds of mortality and 95\% CI are not shown for insignificant variables $(p>0.05)$

${ }^{\dagger}$ Multivariate logistic regression

$30.6 \%, p<0.001$ ), longer hospital LOS (8.9 vs. 8.1 days, $p<0.001)$, longer ICU LOS (3.7 vs. 2.7 days, $p<0.001$ ) and longer ventilator days ( 1.1 vs. 0.5 days, $p<0.001)$ than young patients. In addition to PSM, an MLR analysis showed that in 41,880 patients with BAT, age itself serves as an independent risk factor for mortality (odds ratio: $1.049,95 \%$ CI 1.043-1.055, $p<0.001)$ after adjusting for sex (male), SBP, pulse, RR, GCS, transportation time, ED time, age-associated comorbidity, abdominal surgery, the use of transfusion, and the ISS. However, age-associated comorbidity did not affect mortality significantly in patients with BAT (Table 2).

\section{Effects of age and age-associated comorbidities on outcomes of elderly BAT patients}

In the current study, there were 6216 elderly BAT patients, and $4945(79.6 \%)$ of them had age-associated comorbidities. There was no significant difference in the mortality rate between patients with and those without age-associated comorbidities $(8.2 \%$ vs. $7.1 \%, p=0.718)$. The MLR analysis for this subgroup showed that mortality increased $7.6 \%$ with each year of increasing age after adjustment for the covariables (95\% CI 1.060-1.092, $p<0.001)$, whereas age-associated comorbidity did not affect mortality significantly among elderly BAT patients $(p=0.090)$ (Table 4$)$.

\section{Discussion}

Geriatric trauma constitutes an increasingly recognized problem. Previous literature indicates that the outcomes of trauma in elderly patients are poorer than those in younger patients $[2-4,19,20]$. In this nationwide study, the mortality rate of patients with BAT increased with age, even though the patient number and the ISS decreased with age (Fig. 2). While we separated our patients at the traditional Medicare age of 65 , it is clear that the average age of the elderly bracket was more than double that of the younger patients (75.1 years old vs. 33.9 years old) (Table 1). This would have the effect of accentuating differences in prognosis.

Aging serves as an important risk factor for most diseases and treatments. The health span is usually limited accordingly. The worse outcomes of elderly patients may be due to poorer physiological function, poorer response to stress and more age-associated comorbidities [2-4]. Elderly patients may have an age-related biological vulnerability to trauma insults or diseases. Several studies have indicated that aging is a disease rather than a natural process only $[5,6]$. On the other hand, age-associated comorbidities such as respiratory, cardiovascular or neurological diseases may also worsen trauma outcomes [21,22]. The medications that are usually prescribed for elderly patients may blunt hemorrhage-related hemodynamic changes (vasoactive medications) or increase the risk of hemorrhage (anti-coagulant agents) [23].

In the current nationwide study, elderly patients had significantly poorer outcomes than younger patients under a similar condition and proportion of age-associated comorbidities after a well-batched PSM analysis (Table 1). The risk-adjusted MLR model also showed that age served as an independent risk factor for mortality, but age-associated comorbidity did not (Table 2). The role of age-associated comorbidities seemed insignificant with regard to the mortality of patients with BAT. After controlling for other covariables, patients with BAT mortality increased by $4.9 \%$ with each year of increasing age in patients with BAT. Furthermore, the comparisons of elderly BAT patients with and without age-associated comorbidities showed that there was no significant difference in mortality between these two groups of patients (Table 3). Patients with age-associated comorbidities were older and had lower ISS than patients without age-associated comorbidities. These differences were too small (age: 75.4 vs. 73.9 , ISS: 13 vs. 14) for only statistical significance but not clinical significance. Furthermore, the MLR model showed that age was an independent risk factor for mortality in these patients, but the age-associated comorbidities were not after adjusting for the 
Fig. 2 The distribution of patients with blunt abdominal trauma among ages (lower area), the relationship between the injury severity score and age (middle area) and the relationship between the mortality rate and age (upper area)
Mortality rate (\%)

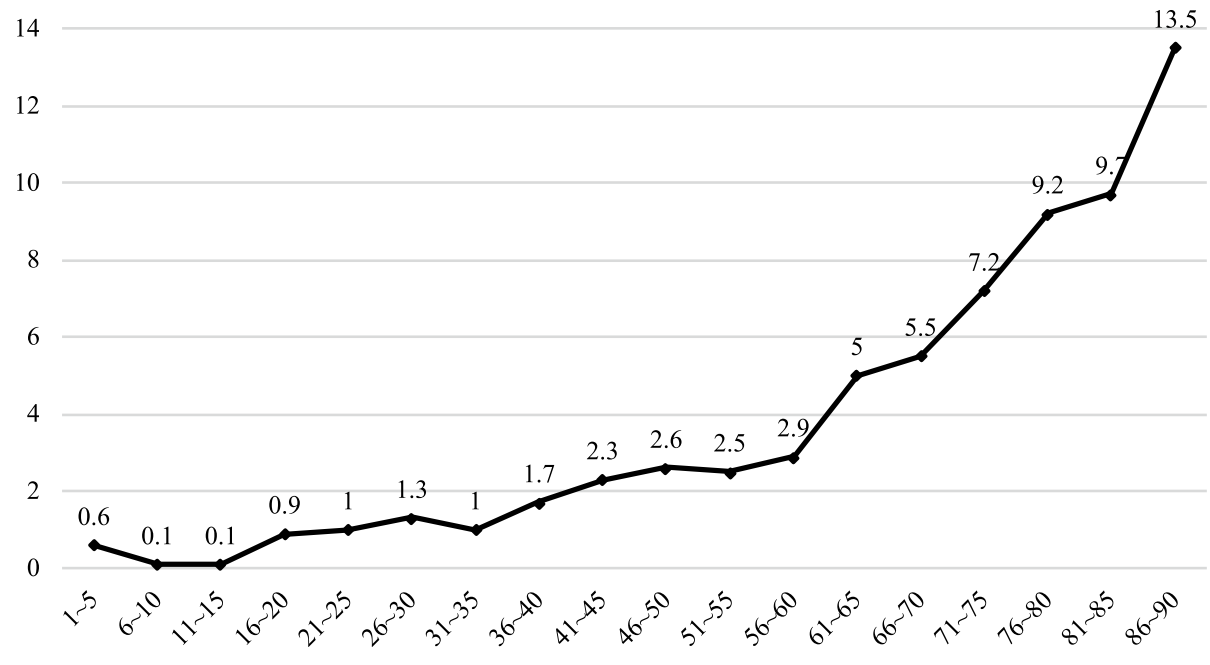

Injury severity score (median with interquartile range)

16

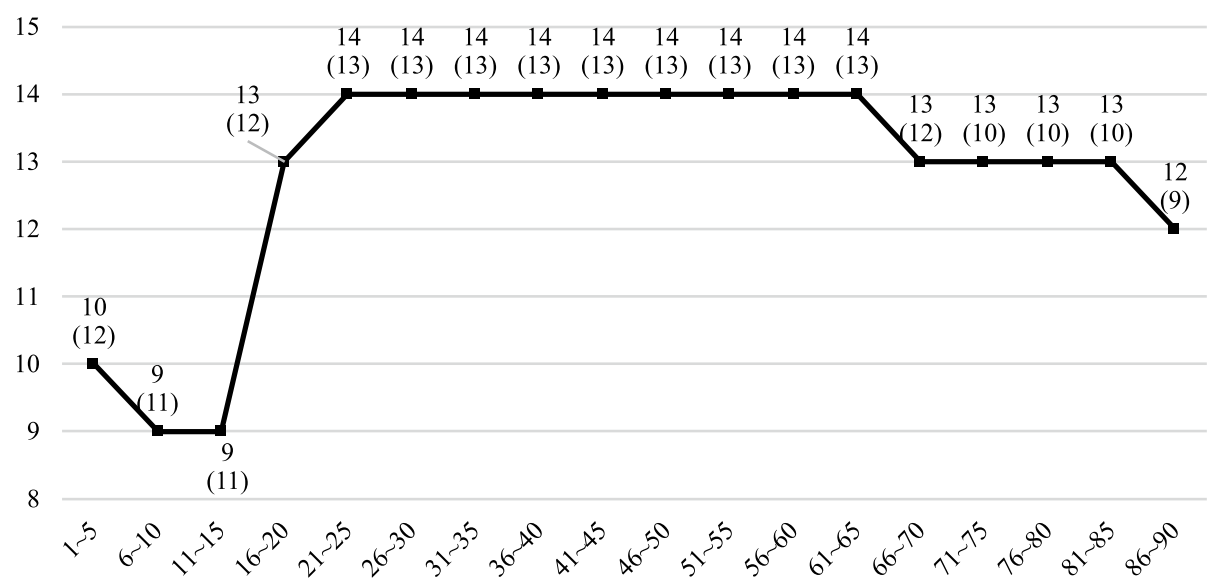

Patient number (N)

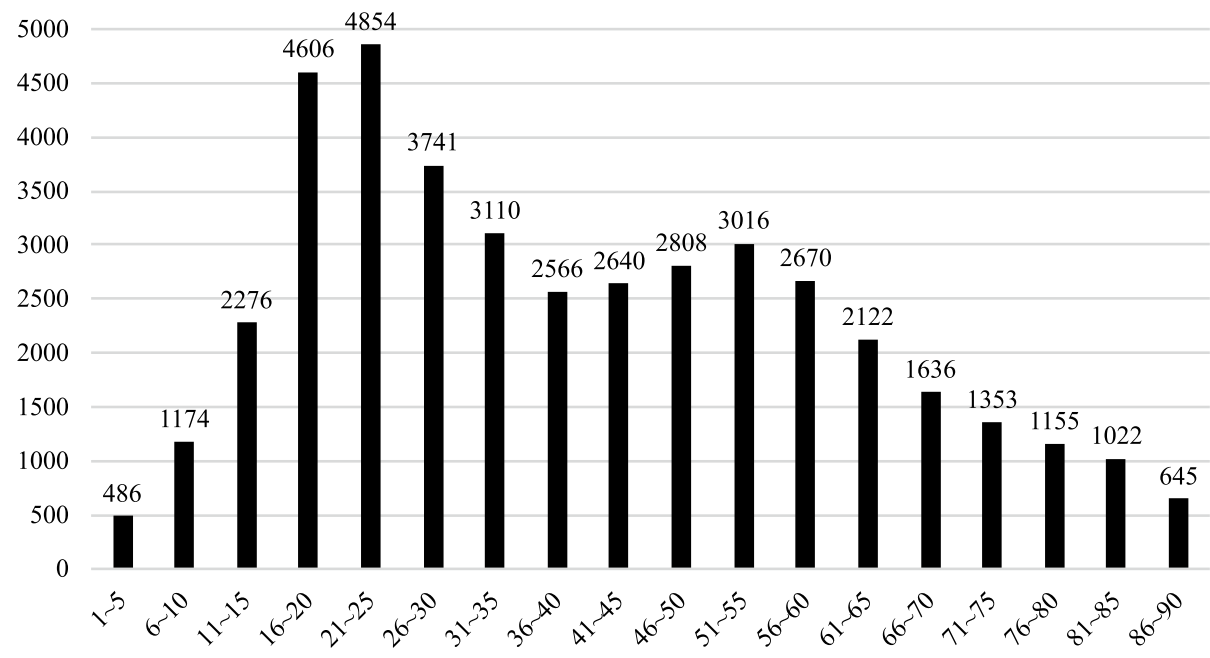

Age of patients with blunt abdominal trauma 
above covariables, which may be significant in univariate analysis (Table 4). The results of the above three analytic methodologies revealed that age itself served as a risk factor for poor outcomes, but age-associated comorbidities did not. The independent effect of age was unrelated to measurable comorbidities in older trauma patients. This might have to do with the cumulative effect of physiological weakening of several organs that have not yet risen to the level of defined comorbidities in the elderly patient. Therefore, the primary target of the management of elderly trauma patients is to combat aging instead of any single age-associated comorbidity.

In the current nationwide study, an inverse trend in injury severity between young and elderly patients was observed. Elderly trauma patients usually have low-energy trauma mechanisms because of their longer home-stay times and lower exposure to risks from work than young patients. The most frequent mechanism of trauma among elderly people is falls from standing height or lower, such as from a commode, bed or wheelchair. The next most common trauma mechanism is motor vehicle collisions. Although elderly patients do not engage in high-risk activities, low-speed crashes may still cause injuries to this population with low physiological reserves. These mechanisms account for up to one-quarter (26.8\%) of geriatric traumas [24, 25]. In addition, elderly patients usually undergo blunt rather than penetrating trauma, which accounts for less than $5 \%$ of elderly trauma patients [26]. Skeletal injuries contributed by osteoporosis are common among elderly patients [27]. Extremity fractures are mostly coded as minor or moderate injuries by the abbreviated injury scale (AIS) system (AIS $=1$ or 2 ) [28]. The above reasons could explain why the ISS increased with age in the young patients as opposed to in the elderly patients (Fig. 2).

On the other hand, even with decreased injury severity, elderly patients had increased mortality with age. The morbidity and mortality seem to be correlated with a geriatric syndrome called frailty, which was defined as multisystem dysregulation yielding decreased physiological reserves and increased vulnerability to stressors [29]. Previous reports indicated that elderly patients may have severe injuries caused by low-speed vehicles and have a doubled mortality rate compared with their younger counterparts [30]. Furthermore, antiplatelets or anticoagulants, which are frequently taken by elderly patients, may increase the probability of hemorrhage in even mild injuries [31]. These facts have implications for how elderly trauma patients should be triaged and treated. Elderly trauma patients are usually undertriaged at trauma centers. Causes for this include relative lower-energy mechanisms of injury, near-normalappearing vital signs and the use of medications that can mask the physiological response to injury. Thus, aggressive treatment and high-level care of geriatric trauma patients might improve outcomes [32].

There are several limitations in our study. The NTDB data are retrospective, and some data were missing in the databank. The records can also be inaccurate. In addition, causal inferences between specific comorbidities and outcomes could not be derived. Statistically, a PSM limitation is that it cannot estimate interactions between covariates and the effects of other confounders. However, we used a greedy (nearest neighbor) approach and a caliper of 0.1 to control the quality to ensure matching adequacy. Then, the standard mean differences indicated balanced matching. The role of age-associated comorbidities in patients with BAT will need more analysis. Further studies with a prospective design and more analyses are needed.

\section{Conclusions}

In patients with BAT, age itself appeared to have an independent and deleterious effect on mortality, but age-associated comorbidity did not. The target for the management of elderly trauma patients is to combat aging instead of combatting any single age-associated comorbidity.

Author contributions Study concept and design: FBokhari, FCY, FBajani. Acquisition of data: FCY. Analysis and interpretation of data: FCY, FBajani, FBokhari, MB, FS. Drafting of the manuscript: FCY, FBajani. Critical revision of the manuscript for important intellectual content: TM, MK, AD, VS, JM, SP.

Availability of data and materials National Trauma Data Bank.

Code availability R software, version 3.5.0 of R Core Team (R Foundation for Statistical Computing, Vienna, Austria, 2018) and R Studio software, version 1.1.453 of R Studio: Integrated Development for R (R Studio, Inc., Boston, Massachusetts, 2016).

\section{Compliance with ethical standards}

Conflict of interest The authors have no commercial associations or sources of support that might pose a conflict of interest.

Ethics approval The current study was approved by the institutional research board of our institution (Ref No. 18-068X).

\section{References}

1. National Center for Health Statistics, National Hospital Ambulatory Medical Care Survey. 2008 emergency department summary tables. http://www.cdc.gov/nchs/data/ahcd/nhamcs_emerg ency/2008_ed_web_tables.pdf. Accessed 31 Aug 2020.

2. Lonner JH, Koval KJ. Polytrauma in the elderly. Clin Orthop Relat Res. 1995;318:136-43.

3. Banks SE, Lewis MC. Trauma in the elderly: considerations for anesthetic management. Anesthesiol Clin. 2013;31(1):127-39. 
4. Frankenfield D, Cooney RN, Smith JS, Rowe WA. Age-related differences in the metabolic response to injury. J Trauma. 2000;48(1):49-57.

5. The Lancet Diabetes Endocrinology. Opening the door to treating ageing as a disease. Lancet Diabetes Endocrinol. 2018;6(8):587.

6. Gladyshev TV, Gladyshev VN. A disease or not a disease? Aging as a pathology. Trends Mol Med. 2016;22(12):995-6.

7. Cimbanassi S, Chiara O, Leppaniemi A, Henry S, Scalea TM, Shanmuganathan K, et al. Nonoperative management of abdominal solid-organ injuries following blunt trauma in adults: results from an International Consensus Conference. J Trauma Acute Care Surg. 2018;84(3):517-31.

8. Madni TD, Ekeh AP, Brakenridge SC, Brasel KJ, Joseph B, Inaba $\mathrm{K}$, et al. A comparison of prognosis calculators for geriatric trauma: a prognostic assessment of life and limitations after trauma in the elderly consortium study. J Trauma Acute Care Surg. 2017;83(1):90-6.

9. https://www.facs.org/quality-programs/trauma/tqp/center-progr ams/ntdb. Accessed 31 Oct 2020.

10. Ferraris VA, Ferraris SP, Saha SP. The relationship between mortality and preexisting cardiac disease in 5,971 trauma patients. $\mathrm{J}$ Trauma. 2010;69(3):645-52.

11. Arhinful E, Jenkins D, Schiller HJ, Cullinane DC, Smoot DL, Zielinski MD. Outcomes of damage control laparotomy with open abdomen management in the octogenarian population. J Trauma. 2011;70(3):616-21.

12. Ferro TN, Goslar PW, Romanovsky AA, Petersen SR. Smoking in trauma patients: the effects on the incidence of sepsis, respiratory failure, organ failure, and mortality. J Trauma. 2010;69(2):308-12.

13. https://www.who.int/healthinfo/survey/ageingdefnolder/en/. Accessed 31 Aug 2020.

14. Austin PC. An introduction to propensity score methods for reducing the effects of confounding in observational studies. Multivar Behav Res. 2011;46(3):399-424.

15. Austin PC, Jembere N, Chiu M. Propensity score matching and complex surveys. Stat Methods Med Res. 2018;27(4):1240-57.

16. Austin PC. Balance diagnostics for comparing the distribution of baseline covariates between treatment groups in propensity-score matched samples. Stat Med. 2009;28(25):3083-107.

17. Anderson RP, Jin R, Grunkemeier GL. Understanding logistic regression analysis in clinical reports: an introduction. Ann Thorac Surg. 2003;75(3):753-7.

18. R Core Team (2014). R: a language and environment for statistical computing. R Foundation for Statistical Computing, Vienna, Austria. http://www.R-project.org/. Accessed 31 Aug 2019.
19. Adams SD, Holcomb JB. Geriatric trauma. Curr Opin Crit Care. 2015;21(6):520-6.

20. Kozar RA, Arbabi S, Stein DM, et al. Injury in the aged: geriatric trauma care at the crossroads. J Trauma Acute Care Surg. 2015;78(6):1197-209.

21. Pandharipande P, Cotton BA, Shintani A, Thompson J, Pun BT, Morris JA Jr, et al. Prevalence and risk factors for development of delirium in surgical and trauma intensive care unit patients. J Trauma. 2008;65(1):34-41.

22. Baijens LW, Clavé P, Cras P, Ekberg O, Forster A, Kolb GF, et al. European Society for Swallowing Disorders-European Union Geriatric Medicine Society white paper: oropharyngeal dysphagia as a geriatric syndrome. Clin Interv Aging. 2016;11:1403-28.

23. Blot S, Koulenti D, Dimopoulos G, Martin C, Komnos A, Krueger WA, et al. Prevalence, risk factors, and mortality for ventilatorassociated pneumonia in middle-aged, old, and very old critically ill patients. Crit Care Med. 2014;42(3):601-9.

24. Gowing R, Jain MK. Injury patterns and outcomes associated with elderly trauma victims in Kingston Ontario. Can J Surg. 2007;50(6):437-44.

25. Braun BJ, Holstein J, Fritz T, Veith NT, Herath S, Mörsdorf P, et al. Polytrauma in the elderly: a review. EFORT Open Rev. 2017;1(5):146-51.

26. Labib N, Nouh T, Winocour S, Deckelbaum D, Banici L, Fata P, et al. Severely injured geriatric population: morbidity, mortality, and risk factors. J Trauma. 2011;71:1908-14.

27. Roth T, Kammerlander C, Gosch M, Luger TJ, Blauth M. Outcome in geriatric fracture patients and how it can be improved. Osteoporos Int. 2010;21:S615-9.

28. Jørgensen K. Use of the abbreviated injury scale in a hospital emergency room. Potential for research in accident epidemiology. Acta Orthop Scand. 1981;52(3):273-7.

29. Fried LP, Ferrucci L, Darer J, Williamson JD, Anderson G. Untangling the concepts of disability, frailty, and comorbidity: implications for improved targeting and care. J Gerontol A Biol Sci Med Sci. 2004;59(3):255-63.

30. Bonne S, Schuerer DJ. Trauma in the older adult: epidemiology and evolving geriatric trauma principles. Clin Geriatr Med. 2013;29(1):137-50.

31. Whitlock EP, Burda BU, Williams SB, Guirguis-Blake JM, Evans $\mathrm{CV}$. Bleeding risks with aspirin use for primary prevention in adults: a systematic review for the US Preventive Services Task Force. Ann Intern Med. 2016;164(12):826-35.

32. Menaker J, Scalea TM. Geriatric care in the surgical intensive care unit. Crit Care Med. 2010;38(9 Suppl):S452-9. 